\title{
Self-perception of surface hydration effect on teachers' voice quality: an intervention study
}

Emile Rocha Santana(1)

Tânia Maria de Araújo(2)

Maria Lúcia Vaz Masson ${ }^{(3)}$

(1) Universidade Estadual da Bahia - UNEB, Salvador, Bahia, Brasil.

(2) Universidade Estadual de Feira de Santana - UEFS, Feira de Santana, Bahia, Brasil.

(3) Universidade Federal da Bahia - UFBA, Salvador, Bahia, Brasil.

Research support sources: 1- Fundação de Amparo à Pesquisa do Estado da Bahia -Fapesb. Public Notice 028/2012: Project

"Teaching Work and Health Conditions: interventions for building healthy work environments." (Process 132/2013);

2- Conselho Nacional de Desenvolvimento Científico e Tecnológico - CNPq. Public

Notice Universal MCTI no. 14/2014: Project

"Protective Strategies for Dysphonia in

Teachers" (Process: 458053/2014-7).

Conflict of interests: Nonexistent

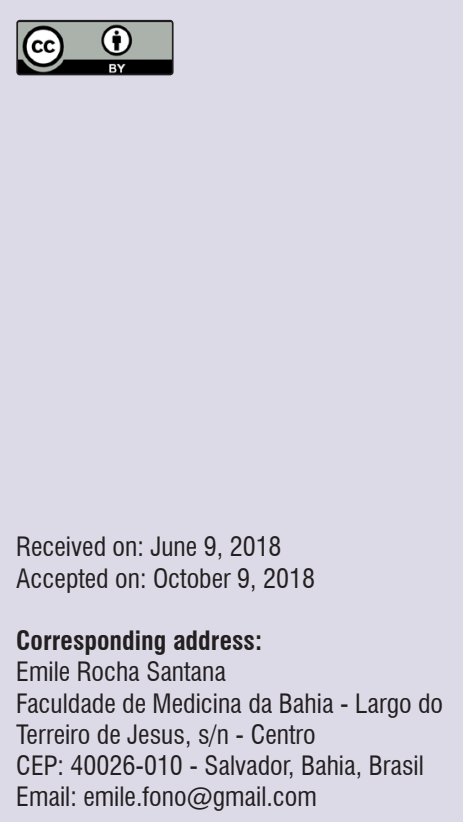

\section{Abstract}

Objective: to assess the effects of surface hydration on vocal quality according to teachers' self-perception.

Methods: an examiner-blinded pre-test and post-test intervention study carried out with a single group of 27 teachers. Sociodemographic characteristics and work questionnaire, Screening Index for Voice Disorder, Vocal Severity Rating Scale, Expected Effects Pre-intervention, Perceived Effects Post-intervention and Post-intervention Perception instruments, were applied. The intervention lasted 4 weeks and consisted of 5 minutes of nebulization with saline solution (sodium chloride $0.9 \%$ ), after 10 minutes of mouth dehydration, prior to teaching.

Results: screening Index for Voice Disorder scores demonstrated a statistically significant reduction $(p=0.001)$. Frequency of voice disorders in the Vocal Severity Rating Scale was reduced without a statistical significance $(p=0.172)$. Expected Effects, Pre-intervention, and Perceived Effects, Post-intervention, showed improvement in vocal quality $(40.7 \%)$, vocal comfort $(40.7 \%)$ and vocal folds hydration (37\%). Post-intervention perception form showed moderate improvement of the symptoms (59.3\%), cleared voice (55.6\%) and ease speaking (48.1\%) as well as intense belief in the intervention (74.1\%) and intense compliance to the intervention (74.1\%).

Conclusion: surface hydration with saline solution promoted self-perceived improvement in vocal quality.

Keywords: Occupational Health; Faculty; Voice Disorders; Self-Assessment; Respiratory Therapy 


\section{INTRODUCTION}

Communication plays a significant role in teachers' professional performance. As such, a voice disorder may have broad implications on teachers' quality of life and work'.

Vocal problems are common among teachers ${ }^{2-5}$. The majority of teachers overload their voices because of loud speaking for long periods without enough time for vocal recovery ${ }^{3}$.

The association between prolonged voice use and work-related, environmental, and individual factors contributes to the prevalence of vocal complaints ${ }^{6,7}$. Once such complaints are identified as vocal deviations associated with professional activity, they are classified as Voice Disorders Related to Work (VDTW) ${ }^{4,8}$.

Research has shown that dysphonia among teachers may be associated with work environment and organization, psychosocial issues, gender and individual factors ${ }^{1,5-8}$.

In studies comparing teachers and non-teachers in the United States and Brazil, significantly higher occurrence of vocal symptoms were observed among teachers, as well as multiple episodes of dysphonia, increased reporting of vocal disturbance and association of these symptoms with professional voice use $e^{2,7}$. Moreover, a higher rate of work absence throughout the year was observed among teachers due to dysphonia, as well as a greater tendency of limitations on their ability to perform tasks and reduction of interaction with others ${ }^{2}$.

Although teachers are the category of voice professionals most researched in the world, few intervention studies are available, especially in regard to prevention of vocal injuries in this population. The limited number of intervention studies has been a major obstacle to speech therapy and to elaborating measures of vocal health promotion, prevention, and public policies ${ }^{9}$.

Hoarseness, vocal fatigue and throat dryness are the symptoms most often reported by these professionals ${ }^{4,5,8}$.

An individual's self-assessment of his or her vocal problem and the result analysis of specific treatments are means used to verify the effectiveness of an intervention and to develop procedures for clinical practice and preventive actions ${ }^{7}$.

A study that evaluated the correlation between three components of vocal disorder diagnosis in teachers (i.e., self-report, perceptual evaluation and acoustic analysis) concluded that self-report was considered an excellent predictor of vocal alteration, with good level of agreement with perceptual assessment ${ }^{10}$. Therefore, self-report measurements constitute a valid method of reliable performance to identify vocal conditions.

Surface or systemic hydration can be considered a good contributing measure of voice protection. Hydration is responsible for promoting vocal efficiency by reducing phonation effort and relieving symptoms and discomforts associated with laryngeal dryness and viscous secretion ${ }^{11}$.

A study examining the effects of three nebulizer treatments on Phonation Threshold Pressure (PTP) and Perceived Phonatory Effort (PPE) used substances with different osmotic properties: saline solution isotonic $0.9 \% \mathrm{NaCl}$, hypertonic saline - $7 \% \mathrm{NaCl}$, and sterile water (hypotonic solution) indicated that inhalation with isotonic saline solution showed more advantageous effects than with the latter two. This was probably because saline solution facilitated short-term laryngeal hydration without altering the ionic balance of the system and of the physiological superficial tissue hydration mechanism in healthy individuals ${ }^{12}$.

A Brazilian research that aimed to investigate the effects of surface hydration on teachers' voice quality with saline solution isotonic $0.9 \% \mathrm{NaCl}$ showed improvement on some teachers' acoustics parameters such as fundamental frequency and intensity parameters ${ }^{13}$.

Another Brazilian study that aimed to compare the effects of two protective strategies - voice amplification (VA) and $0.9 \% \mathrm{NaCl}$ nebulization (NEB) - on teachers' voice in the work setting showed that VA and NEB may help mitigate dysphonia in different pathways, being potential interventions for protecting teachers' voices ${ }^{14}$.

The purpose of this article is to verify the effects of surface hydration with saline solution $(0.9 \% \mathrm{NaCl})$ on teachers' voice quality according to their self-reported perception, in order to assess whether hydration can positively affect vocal health when teaching.

\section{METHODS}

This study was was registered in the Brazil Platform, under CAAE No. 19722913.4.0000.0053 and approved by the Research Ethics Committee of the State University of Feira de Santana (UEFS) [Universidade Estadual de Feira de Santana] as part of the Project "Teaching Work and Health Conditions: interventions for building healthy work environments" (supported by FAPESB) and "Protective Strategies for Dysphonia in Teachers" (supported by CNPq) under report No. 423.012 and compliant with ethical aspects 
in accordance with Resolution 466/12 of the National Council of Health [Conselho Nacional de Saúde].

\section{Design and Population}

This was an examiner-blind pre- and post-test intervention study, with one group of subjects. The study included twenty-seven (27) public school teachers from the city of Salvador, Bahia, Brazil, between September and November of 2014. Subjects were randomly selected.

The school investigated was located in a lower socioeconomic area of Salvador. The school had 72 teachers in total who were responsible for the education of more than two thousand students, including primary, secondary and technical levels. All teachers were invited to take part in this study and 36 subjects agreed to participate.

Inclusion criteria were that teachers had to work a minimum of 20 hours per week and use their voices professionally only as teachers. Teachers were excluded from the study if they presented with any upper respiratory infection (URI) on the days of the recordings, or undergoing speech therapy at the time of the study, or did not participate in all stages of the research.

Exclusions of subjects from the study included end of contract from the institution or retirement (2 cases), health issues during the stages of the intervention (2 subjects), undergoing speech therapy alongside the intervention (1 subject) and failure to participate in all stages of the study (4 subjects).

\section{Study Phases}

\section{Presentation and Informed Consent Form}

The consent term was signed by the school's director. Afterwards, the field research began and teachers were approached across their Complementary Activity (AC) shifts, presented with the project, and invited to take part in the study. Teachers who had shown interest in participating in the research were given an envelope containing two copies of the Informed Consent Form (ICF) (one for the subject and the other for the researcher) and a structured questionnaire developed by the research team.

The questionnaire included sociodemographic questions, and questions related to general characteristics of the work environment and work organization, habits, lifestyle, evaluation of vocal diseases, musculoskeletal disorders, and mental health. The goal was to characterize the population according to these aspects.

\section{Voice Self-Assessment}

Voice self-assessment was carried out pre- and post-intervention by the "Screening Index for Voice Disorder", developed and validated by Ghirardi et al. ${ }^{15}$, and by the "Vocal Severity Rating Scale" based on Roy et al. ${ }^{3}$.

Additional questionnaires utilized were the "Expected Effects Pre-intervention" and "Perceived Effects Post-intervention", both prepared by the staff, and the "Post-Intervention Perception" adapted from Roy et al. ${ }^{3}$.

The Screening Index for Voice Disorders (SIVD) is an efficient tool with high sensitivity for screening of voice disorders in teachers.

This instrument consists of 12 vocal symptoms: hoarseness, voice loss, voice breaks, low-pitched voice, phlegm, dry cough, cough with secretion, pain when speaking, pain when swallowing, secretion/phlegm in throat, dry throat, and strained speech. Each symptom is rated as never, sometimes, almost always, or always. The SIVD score is obtained by summing the number of symptoms reported "almost always" and "always", each counting as one point. The cutoff point is 5 , which determines the level of suspicion for dysphonia ${ }^{15}$ (i.e., scores below 5 indicate no dysphonia).

The Voice Severity Rating Scale (VSRS) classifies the severity of vocal alteration resulting from the subjects' self-report. This instrument consists of a Likert scale of four points $(0=$ no problem, $1=$ mild problem, $2=$ moderate problem and $3=$ severe problem $)^{3}$.

The forms "Expected Effects Pre-intervention" and "Perceived Effects Post-intervention" consisted, respectively, of the following open sentences: "In your opinion, what effects do you expect?", and "what effects did you experience?"

The instrument "Post-intervention Perception" allowed for an objective evaluation of teachers' perception and improvements in communication. The teachers rated on a Likert scale ("Not at all/Very Little", "Moderate" and "Intense") how much improvement was noted in terms of vocal quality, voice clarity, ease of speaking, belief in intervention, and their level of compliance to the intervention. 


\section{Intervention}

All members of the research team received previous training through workshops, to ensure standardization of preparation procedures, equipment hygiene, and monitoring of teachers throughout the surface hydration with saline solution ( $\mathrm{NaCl} 0.9 \%$ ) intervention period.

Similarly, all teachers included in the study underwent an initial presentation of equipment and received information about the preparation procedures and hygiene intervention in order to have access to all information on the conduct laid down in intervention.

Prior to teaching, teachers were submitted to dehydration of the vocal tract mucosa via a 10-minute period of oral breathing, using a nose clip to occlude the nostrils, according to the procedures recommended by the literature ${ }^{12,16,17}$. Surface hydration consisted of inhalation of $5 \mathrm{ml}$ of saline solution $(0.9 \% \mathrm{NaCl})$ for five minutes using the NS Evolusonic ultrasonic nebulizer device. Surface inhalation intervention took place for four weeks during the teachers' longest working shifts.

Each participant was provided with his/her own nebulizer that was allotted a code in advance. All inhalations were followed up and monitored by the research team daily.

Two inhalations were planned for the teachers in the morning and afternoon shifts and a single inhalation for those on the night shift. During daytime shifts (4 hours and 10 minutes total), the first inhalation was conducted prior to start of the first class, and the second during their break, which was two hours after the first class. This average time frame of two hours was adopted because suggested by short-duration studies on surface hydration to be the time required for the prejudicial viscosity of the vibration of the vocal folds to return ${ }^{16}$. The teachers on the night shift underwent a single inhalation session prior to the beginning of their first class, since the workload in this shift was lower (2 hours and 20 minutes total with no break).

\section{Data analysis}

The differences between the pre- and postintervention values of the SIVD total score, VSRS, "Expected Effects Pre-intervention", "Perceived Effects Post-intervention", and "Post-intervention Perception" were analyzed.

The Statistical Package for the Social Sciences SPSS version 19.0 for Windows, software package was used for data storage and analysis. The non-parametric Wilcoxon Signed-rank test was used to compare samples pre- vs. post-intervention, and the McNemar's test for paired samples to compare paired nominal data pre- vs. post-intervention. A statistical significance level of $5 \%(p \leq 0.05)$ was adopted for all tests. Absolute frequencies calculations were used as well.

Data on "Expected Effects Pre-intervention" and "Perceived Effects Post-intervention" were collected as open sentences. The evaluation of the responses was done using content analysis. Content analysis is a technique used to identify repeated units of a text, phrases or words, establishing a category that represents them ${ }^{18}$. Thus, the material collected was submitted to reading and rereading, proceeding to the identification of key categories capable of expressing the obtained reports. Based on this analysis, it was possible to identify the several types of reported effects and assess their frequency. Then the most mentioned effects were reported, which allowed for the comparison of the main effects mentioned in the preand post-intervention.

\section{RESULTS}

\section{Sample and Working Environment Characterization}

After applying the exclusion criteria, the population of this study consisted of 27 teachers, 15 females and 12 males, with a mean age of 44.9 years and average of 18 years of teaching. The average weekly work hours as a teacher was 28.5 hours in the school in which the intervention was performed. The majority said that their workplace had unfavorable acoustic (70.4\%), noisy rooms $(78.8 \%)$, adequate temperature $(55.6 \%)$ and without humidity $(77.8 \%)$ with an average of 39.6 students per class.

In regard to aspects of vocal health, it was observed that $55.6 \%$ of teachers reported consuming some form of alcoholic beverage; $74.1 \%$ reported loud talking in class and $32 \%$ mentioned shouting while teaching; $51.9 \%$ reported drinking water while teaching, with 6.3 average glasses of water consumed per day. It was found that $29.6 \%$ reported having vocal alteration and $11.1 \%$ said they have already been absent from work due to a voice issue.

\section{Vocal Self-Assessment}

The SIVD total score comparison pre- and postintervention showed a statistically significant reduction $(p<0.05)$. 
Vocal Severity Rating Scale (VSRS), showed reduction for self-reported moderate dysphonia frequency and severe dysphonia frequency and increasing for mild dysphonia without statistical significance $(p=0.172)$

Table 1. Pre vs. post means and standard deviations comparison of the Screening Index for Voice Disorder (SIVD) of 26 teachers from public state school. Salvador, Bahia, Brazil, 2014

\begin{tabular}{cccc}
\hline \multirow{2}{*}{ SIVD } & \multicolumn{3}{c}{$\begin{array}{c}\text { Hydration }(\mathrm{NaCl} \mathrm{0,9 \% )} \\
(\mathbf{N}=\mathbf{2 6})\end{array}$} \\
\cline { 2 - 4 } & Mean & SD & p-value \\
\cline { 2 - 4 } Pre & 3.50 & 2.97 & $\mathbf{0 . 0 0 1}^{\mathrm{a}}$ \\
Post & 1.23 & 2.03 & \\
\hline
\end{tabular}

aWilcoxon Signed-rank test

* The scores of 26 individuals were analyzed, due to the incorrect filling of the protocol by one of the teachers.

$\mathrm{SD}=$ Standard Deviation

$\mathrm{NaCl}=$ Sodium Chloride

Table 2. Pre- vs. post intervention comparison of the Vocal Severity Rating Scale (VSRS) frequencies, of 26 teachers from public state school. Salvador, Bahia, Brazil, 2014

\begin{tabular}{cccc}
\hline Variable & $\begin{array}{c}\text { Hydration Group(N= 26) } \\
(\mathbf{N}=\mathbf{2 6})\end{array}$ \\
\hline Vocal Quality & Pre \%(n) & Post \%(n) & p-value \\
\hline Normal & $52.0(13)$ & $44.4(12)$ & \\
Mild Dysphonia & $28.0(7)$ & $44.4(12)$ & $0.172^{\mathrm{b}}$ \\
Moderate Dysphonia & $20.0(5)$ & $11.1(3)$ & \\
\hline
\end{tabular}

'McNemar's test for paired samples

* Severe dysphonia was reported by one subject only during pre-intervention. This result had to be removed from the calculation, so as to make possible the application of the statistical test.

$\mathrm{NaCl}=$ Sodium Chloride

"Expected Effects Pre-intervention" revealed that "improvement in voice quality," "vocal comfort" and "hydration of the vocal folds" were the most frequent effects mentioned by teachers. The "Perceived Effects on post-intervention" revealed a slight reduction of "improved voice quality" and increased frequency of "vocal comfort" and "hydration of the vocal folds"
The perception of the intervention form showed there was a higher "moderate" frequency for: improvement of vocal symptoms, clearer voice and ease of speaking. Most teachers rated their belief in the intervention as "intense", thus reporting good compliance to the intervention 
Table 3. Frequency of Expected Effects pre-intervention and Perceived effects post-intervention of 27 teachers from public state school. Salvador, Bahia, Brazil, 2014

\begin{tabular}{lcccc}
\hline \multirow{2}{*}{ Variables } & \multicolumn{2}{c}{ Expected Effects Pre-intervention } & \multicolumn{2}{c}{ Perceived Effects Post-intervention } \\
\cline { 2 - 5 } & $\mathbf{n}$ & $\%$ & $\mathbf{n}$ & $\%$ \\
\hline Vocal Quality Improvement & 14 & 51.9 & 11 & 40.7 \\
Vocal Comfort & 8 & 29.6 & 11 & 40.7 \\
Vocal folds Hydration & 8 & 29.6 & 10 & 37 \\
Dysphonia detection & 3 & 11.1 & - & - \\
Prevention of Vocal Problems & 2 & 7.4 & 2 & 7.4 \\
Vocal Projection Improvement & 2 & 7.4 & 2 & 7.4 \\
Breathing Improvement & 2 & 7.4 & 1 & 3.7 \\
Vocal Stability & 1 & 3.7 & 3 & 11.1 \\
Clean Voice & 1 & 3.7 & 3 & 11.1 \\
Elimination of mucous and airway clearance & - & - & 4 & 14.8 \\
Pitch Changing & - & - & 1 & 3.7 \\
Vocal Flexibility & - & - & 1 & 3.7 \\
Easier Speaking & - & - & 1 & 3.7 \\
\hline
\end{tabular}

Table 4. Post-intervention perception of 27 teachers from public state school, Salvador, Bahia, Brazil, 2014

\begin{tabular}{cc}
\hline Variable & $\begin{array}{c}\text { Hydration Group } \\
\text { (N=27) \%(n) }\end{array}$ \\
\hline Improvement of the Symptoms & \\
Not at all/Very Little & $14.8(4)$ \\
Moderate & $59.3(16)$ \\
Intense & $25.9(7)$ \\
\hline Clearer Voice & \\
Not at all/Very Little & $18.5(5)$ \\
Moderate & $55.6(15)$ \\
Intense & $25.9(7)$ \\
\hline Ease Speaking & \\
Not at all/Very Little & $18.5(5)$ \\
Moderate & $48.1(13)$ \\
Intense & $33.3(9)$ \\
\hline Believe in the Intervention & \\
Not at all/Very Little & $0.00(0)$ \\
Moderate & $25.9(7)$ \\
Intense & $74.1(20)$ \\
\hline Self-perceived level of compliance & \\
Not at all/Very Little & $3.7(1)$ \\
Moderate & $22.2(6)$ \\
Intense & $74.1(20)$ \\
\hline
\end{tabular}

\section{DISCUSSION}

\section{Vocal Self-Assessment}

The data for the SIVD total score showed statistically significant reduction after intervention $(p<0.05)$. It was also observed that the SIVD averages were below the risk for the development of voice disorders. This corroborated with the socio-demographic questionnaire in which most teachers $(70.4 \%)$ reported no voice problems.

The reduction of the value of SIVD suggested that surface hydration with saline solution $(0.9 \% \mathrm{NaCl})$ provided significant decrease of vocal symptoms perceived by teachers.

Hydration procedures are considered important in the prevention and treatment of dysphonia, such as dysphonia caused by vocal nodules - the most common voice pathology found in teachers ${ }^{19}$.

Similarly, the Vocal Severity Rating Scale (VSRS) and the "Post-intervention Perception" forms answered by the participants revealed reduction. Although these findings have not shown statistically significant differences, the observed reduction could point to a potentially beneficial effect of the intervention. It is possible that a study with a larger follow-up period could reveal more pronounced changes than what was evidenced in this study's four weeks of observation.

In regards to the Post-intervention Perception form, it was shown that $59.3 \%$ of the subjects have reported moderate improvement in voice symptoms and more than $25 \%$ reported intense improvement. 
These results are consistent with what was observed in SIVD, confirming the reduction of vocal symptoms after surface hydration with saline solution $(0.9 \% \mathrm{NaCl})$.

It is important to remember that, although the majority of teachers in this study had reported not smoking, most $(51.9 \%)$ reported drinking less amount of water than what was indicated (2 liters/day), and a considerable percentage reported drinking some form of alcoholic beverage (55.6\%).

The literature suggests increasing water intake, improving environmental humidification and / or performing steam inhalation to prevent or to treat excessive dryness of the vocal folds ${ }^{11}$.

The Perception Post-intervention form also indicated that the majority of teachers reported moderate to intense for clearer voice and moderate to intense for ease of speaking. These results are consistent with other studies that investigated the Perceived Effects Post-intervention and the effects of surface hydration with saline solution $(0.9 \% \mathrm{NaCl})$ on voice quality ${ }^{12-14,16,17}$.

Expected Effects Pre-intervention and Perceived Effects Post-intervention showed improved voice quality, vocal comfort and hydration of the vocal folds.

Hydration promotes optimization of biomechanical characteristics of the vocal folds, increasing the efficiency of the oscillation due to the decrease in the phonation pressure threshold (PTP). In turn, this may lead to reduced viscosity, improving vocal quality ${ }^{19}$. Furthermore, the perceived vocal effort (PPE), also controlled by subglottic pressure, has an inverse relation with hydration; hence, hydration tends to make voice production easier, whereas dehydration can make voice production forced ${ }^{19,20}$. This helps explain the relationship between surface hydration with saline solution and increase in perceived vocal comfort as well as the reporting of moderate to intense ease of speaking after intervention.

In studies that examined the effects of nebulization with saline solution (saline $0.9 \% \mathrm{NaCl}$ ) and hypotonic solution (sterile water) on PTP and PPE in singers and in subjects with Sjögren's syndrome ${ }^{16,17}$ a better response was observed with saline solution. A similar result was found in a previous study on healthy women ${ }^{13}$, confirming the results of this intervention.

In the current study, the observed findings suggest a possible relationship between voice quality improvement referred as Perceived Effects Post-intervention and the frequency of moderate to intense clearer voice of the Perception Post-intervention instrument. In addition, vocal comfort found in
Perceived Effects Post-intervention may be related to moderate to intense rating of ease of speaking of the Perception Post-intervention instrument. These symptoms are associated with increased hydration of the vocal folds, effect also referred to by the participants in the Perception Post-intervention instrument. Surface hydration with saline solution $(\mathrm{NaCl} 0.9 \%)$ may have possibly decreased the viscosity of the vocal tract mucus, as occurred in other intervention studies ${ }^{20,21}$.

This study investigating inhalation with saline solution differed from other studies investigating the immediate effect using the same substance ${ }^{12,16,17}$. This investigation was configured as a long-term intervention with no immediate effect evaluation, which makes compliance more difficult, requiring therefore, greater control and monitoring. However, it corroborated with the results obtained in those studies demonstrating positive effect of laryngeal hydration with saline solution ( $\mathrm{NaCl} 0.9 \%$ ).

In long-term $0.9 \% \mathrm{NaCl}$ nebulization intervention with teachers, It was observed improvement in some acoustics parameters such as an increase in their mean fundamental frequency (F0) of the vowel /a:/ and reduction on their intensity parameters of connected speech and spontaneous speech. The authors suggested that surface hydration with $0.9 \% \mathrm{NaCl}$ promoted those results because it generated viscosity reduction of the mucus in teachers' vocal folds. That led to a lighter and quicker vibration, reduction in glottal resistance and, phonatory effort ${ }^{13}$.

A four-week intervention that aimed to compare the effects of two protective strategies - voice amplification (VA) and $0.9 \% \mathrm{NaCl}$ nebulization (NEB) - on teachers' voice in the work setting demonstrated that these two strategies might protect the teachers' voice. Both groups (VA and NEB) showed a decrease in sound pressure level (SPL) after the intervention. According to the authors, a decrease in SPL is one of the most crucial factors directly related to vocal overload. This decrease can protect teachers' voices from the cumulative effect seen in professional voice users ${ }^{14}$.

Thus, the intervention results above may suggest that surface hydration with saline solution could be an effective protective measure of voice quality, potentially reducing the occurrence of voice disorders in teachers.

In regard to the compliance to the intervention, the majority $(74.1 \%)$ of participants self-reported compliance as intense. Also, most of the teachers rated belief as intense in the intervention, as evidenced by self-reported findings. 
Findings from this study may lead to the possibility of a replication of this intervention in other schools, with other teachers.

It is worth noting that few studies have been developed on the effects of protective intervention on teachers' vocal quality worldwide. In Brazil, an intervention study was found on hydration with professional voice users. The authors detected via videokymography positive differences in the vibration characteristics of the vocal folds' mucosal wave after combined larynx hydration (surface and systemic) ${ }^{22}$, which corroborates with results of the current study.

Two additional Brazilian intervention studies ${ }^{13,14}$ were developed with $0.9 \% \mathrm{NaCl}$ nebulization over the past years, having teachers as the subject. It was observed improvement on teachers' vocal quality as well as that the nebulization with saline solution can be a potential voice protective measure.

Limitations of the conducted study need to be addressed. First, the small number of participants may have led to distinct types of biases, especially type II error - false negative. However, it was observed that most intervention studies on laryngeal hydration found in the literature also used a small number of subjects ${ }^{11-}$ $14,16,17,19,20$. Another limitation is in regard to potential biases that may have occurred due to difficulties arising from the sampling procedure (random sample). Furthermore, this study was conducted on a daily basis with teachers at school and not in a laboratory, and with no control group for comparison of results. Therefore, analyzes were based on a pre- and post-intervention.

Finally, this research used only teachers' selfperception of the effects of hydration on their voices through self-report forms. However, this should not minimize its importance, as the use of self-assessment forms in research brings the teachers' perspective in several voice-related aspects and problems ${ }^{9}$. Thus, the latter can be considered a positive factor rather than a limitation, and should be incorporated into future studies addressing more objective aspects of the measures investigated.

\section{CONCLUSION}

The surface hydration with saline solution showed benefits for all teachers in the intervention, since an improvement was observed in vocal self-perceived symptoms of the majority, after a four-week intervention. Despite the complexity, controlled studies with larger numbers of teachers with a control group must be performed to confirm the protective effects of the hydration observed in this study.

\section{REFERENCES}

1. Giannini SP, Latorre MR, Fischer FM, Ghirardi AC, Ferreira LP. Teachers' voice disorders and loss of work ability: a case-control study. J. Voice. 2015;29(2):209-17.

2. Roy N, Merril RM, Thibeault S, Gray SD, Smith EM. Voice disorders in teachers and the general population: effects on work performance, attendance, and future career choices. J Speech Lang Hear Res. 2004;47(3)542-51.

3. Roy N, Weinrich B, Gray S, Stemple J, Sapienza C. Three treatment for teachers with voice disorders: a randomized clinical trial. Speech Lang Hear Res. 2003;46(3)670-88.

4. BRASIL. Ministério da Sáude. Secretaria de Vigilância em Saúde. Departamento de Saúde Ambiental e Saúde do Trabalhador. Distúrbio de Voz Relacionado ao Trabalho. Brasília:Ministério da Saúde, 2011. Available at http://aborlccf.org.br/ imagebank/Protocolo_DVRT.pdf . Accessed May 10, 2014.

5. Araujo TM, Carvalho FM. Work conditions and health of teachers in the State of Bahia, Brazil: epidemiological studies. Educ. Soc. 2009;30(107):427-49.

6. Marçal CCB, Peres MA. Self-reported voice problems among teachers. Rev Saúde Pública. 2011;45(3): 503-11.

7. Behlau M, Zambon F, Guerrieri AC, Roy N. Epidemiology of voice disorders in brazilian teachers and nonteachers in Brazil: prevalence and adverse effects. J Voice. 2012;26(5):665e9-e18.

8. Ferreira LP, Martz MLW. Distúrbios da voz relacionados ao trabalho: a experiência dos Cerest. Bepa [peridodico na internet] 2010 [Accessed May 10, 2014];7(76):13-9. Available at:

9. http://www.saude.sp.gov.br/resources/ccd/ homepage/bepa/2010/bepa_76_-_abril_2010.pdf.

10. Dragone MLS, Ferreira LP, Giannini SPP, SimõesZenari M, Vieira VP, Behlau M. Teachers' voice: a review of 15 years of SLP contribution. Rev. Soc. Bras. Fonoaudiol. 2010;15(2):289-96.

11. Jesus LB. Concordância entre diferentes definições operacionais de alteração vocal [Dissertação]. Salvador (BA): Universidade Federal da Bahia; 2013 [acesso em 2013 Out 12]. Available at: http://www. 
sat.ufba.br/site/db/dissertacoes/1972013150452. pdf.

12. Solomon NP, DiMattia MS. Effects of a vocally fatiguing task and systemic hydration on phonation threshold pressure. J. Voice. 2000;14(3):341-62.

13. Tanner K, Roy N, Merrill RM, Elstad M. The effects of three nebulized osmotic agents in the dry larynx. J Speech Lang Hear Res. 2007;50(3):635-46.

14. Santana ÉR, Masson MLV, Araújo TM. The effect of surface hydration on teachers' voice quality: an intervention study. J. Voice. 2017;31(3):383.e5-e11.

15. Masson MLV, Araujo TM. Protective strategies against dysphonia in teachers: preliminaries results comparing voice amplification and $0,9 \% \mathrm{NaCl}$ nebulization. J. Voice. 2018;32(2):257.e1-e10.

16. Ghirardi ACA, Ferreira LP, Giannini SPP, Latorre MRDO. Screening Index for Voice Disorder (SIVD): development and validation. J. Voice. 2013;27(2):195-200.

17. Tanner K, Roy N, Merrill RM, Elstad M, Muntz F, Houtz DR et al. Nebulized isotonic saline versus water following a laryngeal desiccation challenge in classically trained sopranos. J Speech Lang Hear Res. 2010;53(6):1555-66.

18. Tanner K, Roy N, Merrill RM, Kendall K, Miller $\mathrm{KL}$, Clegg DO et al. Comparing nebulized water versus saline after laryngeal desiccation challenge in Sjögren's Syndrome. Laryngoscope. 2013;123(11):2787-92.

19. Caregnato RCA, Mutti R. Qualitative research: discourse analysis versus content analysis. Texto Contexto Enferm. 2006;15(4):79-84.

20. Verdolini-Marston K, Sandage M, Titze IR. Effect of hydration treatments on laryngeal nodules and polyps and related voice measures. J. Voice [peridodico na internet] 1994 [Accessed Aug 12, 2014]; 8(1):30-47. Available at: http://dx.doi. org/10.1016/S0892-1997(05)80317-0.

21. Verdolini-Marston K, Titze IR, Druker DG. Changes in phonation threshold pressure with induced conditions of hydration. J. Voice. 1990;4(2):142-51.

22. Verdolini K, Tizte IR, Fennel A. Dependence of phonatory effort on hydration level. J Speech Lang Hear Res. 1994;37(5):1001-7.

23. Fujita R, Ferreira A, Sarkovas C. Assessment videokymography of mucosal wave in vocal fold before and after hydration. Rev Bras Otorrinolaringol. 2004;70(6):743-8. 